\title{
On Periods for String Compactifications
}

\author{
P. Berglund ${ }^{1}$, E. Derrick ${ }^{2}$, T. Hübsch ${ }^{3}$ and D. Jančić ${ }^{2}$ \\ ${ }^{1}$ School of Natural Science \\ Institute for Advanced Study \\ Olden Lane \\ Princeton NJ 08540 \\ 2 Theory Group \\ Department of Physics \\ University of Texas \\ Austin, TX 78712 \\ ${ }^{3}$ Department of Physics \\ Howard University \\ Washington $D C 20059$
}

\section{ABSTRACT}

Motivated by recent developments in the computation of periods for string compactifications with $c=9$, we develop a complementary method which also produces a convenient basis for related calculations. The models are realized as Calabi-Yau hypersurfaces in weighted projective spaces of dimension four or as Landau-Ginzburg vacua. The calculation reproduces known results and also allows a treatment of Landau-Ginzburg orbifolds with more than five fields.

November 1993

- On leave from the Institute "Ruđer Bošković", Zagreb, Croatia. 


\section{Introduction, Results and Summary}

Until recently, the most general known superstring models with worldsheet $(2,2)$ supersymmetry were also the least amenable to calculation and physics prediction. Such models are built on Calabi-Yau spaces, $\mathcal{M}$, and depend on their complex structure and (complexified) Kähler class: the dynamics of matter fields, respectively the 27's and $27^{*}$ 's of $E_{6}$, is determined by the so-called special geometry of these two sectors of the parameter space ${ }^{1}$ (for a comprehensive review, see [1] and references therein). By a remarkable characteristic of special geometry, both the kinetic and the coupling terms are determined from a single object which is complex analytic over the parameter space $[2,3,4]$. The dynamics of the 27's is governed by the functional dependence over the parameter space of the holomorphic volume-form $\Omega$ on $\mathcal{M}$. It is convenient to express $\Omega$ as a vector over a basis of homology cycles in $\mathcal{M}$ with components $\varpi_{j} \stackrel{\text { def }}{=} \oint_{\gamma^{j}} \Omega$, which are called periods.

At special subregions of the parameter space, the underlying $(2,2)$-superconformal field theory simplifies sufficiently so as to enable effective and accurate calculation of physical data. The generic models, however, remain understood only in terms of their Calabi-Yau geometry, and were widely believed to admit in practice only perturbative calculations of limited accuracy and unlimited difficulty. Certain non-renormalization theorems [5] do ensure the exactness of the $\mathbf{2 7}^{3}$ couplings for which a rather successful technology has developed $[6,7,8]$; using special geometry and the 'mirror map' (for cases where the mirror model is known [9]) then allows the determination of a large portion of the dynamics of such particular models. Finally, combining these facts in an essential way with the complex analyticity over the parameter space has brought forth an approach of hitherto unwitnessed calculational power. First developed on a 1-parameter example [10], and now generalized to a wealth of large families of examples [11], this is absolutely the most widely applicable exact technique.

The results of this article are twofold. Firstly, following a suggestion in Ref. [11], (see also Refs. $[10,12]$ ), we develop a more direct calculation of periods, rather than threading through analytic continuation and modular group action as in Refs. [10,11]. This more direct calculation is applicable to Calabi-Yau spaces, not necessarily of dimension three, described as a single hypersurface in a weighted projective space. More importantly, we are also able to handle string vacua expressed as Landau-Ginzburg orbifolds with more than five fields. The geometrical interpretation of these models is not always known. Nevertheless the periods which we obtain are solutions to a Picard-Fuchs equation whose existence is assured since the models are $(2,2)$ string vacua. Second, as an additional benefit of this approach, a convenient and rather simple homology basis is produced, which allows a simple and intuitive description of periods and is also suitable for topologicalanti-topological fusion calculations [13].

1 In addition, a subset of matter fields, being $1 \mathrm{~s}$ of $E_{6}$, have no Yang-Mills interaction and their dynamics is determined by another, rather less well understood parameter space. 
The paper is organized as follows: in section 2 we describe the technique by which periods are constructed. Section 3 is devoted to some non-trivial examples, while discussions and conclusions are left for section 4 . Some technical details regarding our choice of contours are presented in the appendix.

\section{The Construction}

The Calabi-Yau spaces studied herein will all be defined as complete intersections of hypersurfaces in some (weighted) projective space, each one defined as the zero-set of a defining polynomial $[1,14,15,16,17]$. In fact, we wish to consider families of such spaces, parametrized by the coefficients in the defining polynomials. This only parametrizes the complex structure moduli space. However, using mirror symmetry, this gives us a handle on the Kähler moduli space as well; starting with a mirror pair $(\mathcal{M}, \mathcal{W})$, we study the parameter space of complex structure deformations for $\mathcal{M}$ and $\mathcal{W}$. By the mirror symmetry conjecture this result is the equivalent of the quantum corrected moduli space of the Kähler class corresponding to $\mathcal{W}$ and $\mathcal{M}$ respectively.

Begin with the case of a family of hypersurfaces $\mathcal{M}_{\phi}$ defined as the zero-set of a defining polynomial $P_{\phi}$. The periods are defined as

$$
\varpi_{j}\left(\phi_{\alpha}\right) \stackrel{\text { def }}{=} \int_{\gamma^{j}} \Omega\left(\phi_{\alpha}\right)
$$

where $\Omega\left(\phi_{\alpha}\right)$ is the nowhere vanishing holomorphic 3-form $[6,18]$

$$
\Omega\left(\phi_{\alpha}\right) \stackrel{\text { def }}{=} \operatorname{Res}_{\mathcal{M}_{\phi}}\left[\frac{\left(x \mathrm{~d}^{4} x\right)}{P_{\phi}}\right]
$$

on the Calabi-Yau 3-fold specified by the parameters $\phi_{\alpha}$. The 4-differential $\left(x \mathrm{~d}^{4} x\right)$ is the 'natural' one: on a weighted projective $N$-space $\mathbb{P}_{\left(k_{0}, \ldots, k_{N}\right)}^{N}$, we have

$$
\left(x \mathrm{~d}^{N} x\right) \stackrel{\text { def }}{=} \frac{1}{(N+1) !} \epsilon^{i_{0} i_{1} \cdots i_{N}} k_{i_{0}} x_{i_{0}} \mathrm{~d} x_{i_{1}} \cdots \mathrm{d} x_{i_{N}}
$$

where $k_{i}$ are the weights of the coordinates. That is, in $\mathbb{P}_{\left(k_{0}, \ldots, k_{N}\right)}^{N}$,

$$
\left(x_{0}, \ldots, x_{N}\right) \cong\left(\lambda^{k_{0}} x_{0}, \ldots, \lambda^{k_{N}} x_{N}\right), \quad \lambda \in \mathbb{C}^{*} \text {. }
$$

The period (2.1) will here be calculated by choosing one of the standard coordinate patches in $\mathbb{P}_{\left(k_{0}, \ldots, k_{4}\right)}^{4}, \mathcal{U}_{m}$, where $x_{m} \neq 0$ so that $x_{m}=1$ by projectivity. There [11]:

$$
\varpi_{j}^{(m)}\left(\phi_{\alpha}\right)=C \int_{\Gamma^{j}} \frac{\prod_{i \neq m} \mathrm{~d} x_{i}}{\left[P_{\phi}\right]_{x_{m}=1}},
$$


with $C$ a convenient prefactor. This is easily seen to apply for Calabi-Yau weighted hypersurfaces of arbitrary dimension.

We separate the polynomial into a reference polynomial $P_{0}$ independent of the moduli $\phi$, and a perturbative part $\Delta$ which does depend on the $\phi$. This amounts to choosing a reference point in moduli space, and expanding around that point. The basic idea here is to expand $1 / P_{\phi}$ around $1 / P_{0}$, and utilize the Laplace transform

$$
\frac{1}{P_{\phi}}=\frac{1}{P_{0}-\Delta}=\sum_{n=0}^{\infty} \frac{\Delta^{n}}{P_{0}^{n+1}}=\sum_{n} \Delta^{n} \int_{0}^{\infty} d s \frac{s^{n}}{n !} e^{-s P_{0}}, \quad \Re e\left(P_{0}\right)>0,
$$

which produces a "small- $\phi$ " expansion of the periods (2.5).

With a choice of the poly-contours $\left\{\Gamma^{j}\right\}$ discussed below for the various types of polynomials, Eq. (2.5) may be considered a definition of the periods.

We now discuss suitable contours for all types of reference polynomials found in $[8,19]$. The polynomials are built out of basic patterns labelled Fermat types, tadpoles and loops, which we treat in turn.

\subsection{Fermat Models}

Write the defining polynomial as

$$
P_{\phi}=P_{0}-\sum_{\alpha} \phi_{\alpha} M_{\alpha}
$$

and consider the case where

$$
P_{0}=\sum_{i=0}^{N} x_{i}^{a_{i}}
$$

is the reference polynomial of the Fermat type. The deformation terms,

$$
M_{\alpha}=\prod_{i=0}^{N} x_{i}^{q_{i}^{\alpha}}, \quad \alpha=1, \ldots, M
$$

are suitable monomials. Writing $d=\operatorname{deg}\left(P_{\phi}\right)$, we have that, for homogeneity,

$$
\begin{array}{rlrl}
a_{i} k_{i} & =d, & \forall i \\
\sum_{i} k_{i} q_{i}^{\alpha} & =d, \quad \forall \alpha .
\end{array}
$$

For the hypersurface to be Calabi-Yau, we also need

$$
\sum_{i} k_{i}=d
$$


In the affine coordinate patch $\mathcal{U}_{m}$ where $x_{m}=1$, the defining polynomial becomes

$$
P_{\phi}=1+\sum_{i}^{\prime} x_{i}^{a_{i}}-\sum_{\alpha} \phi_{\alpha} \prod_{i}^{\prime} x_{i}^{q_{i}^{\alpha}}
$$

where the prime on the summation and product sign denotes that the indicated summation and product skip $i=m$. In this patch, and supressing the homology labels, the periods become

$$
\varpi^{(m)}\left(\phi_{\alpha}\right)=C \int_{\Gamma} \mathrm{d}^{4} x \int_{0}^{\infty} \mathrm{d} s \sum_{n=0}^{\infty} \frac{s^{n}\left(\sum_{\alpha} \phi_{\alpha} M_{\alpha}\right)^{n}}{n !} e^{-s P_{0}}, \quad \Re e\left(P_{0}\right)>0 .
$$

The poly-contours $\Gamma$ have to be chosen so that the convergence criterion $\Re e\left(P_{0}\right)>0$ is obtained. To this end, label the poly-contours $\Gamma$ by the $N$-vector $\left(\delta_{0}, \ldots, \widehat{\delta}_{m}, \ldots, \delta_{N}\right)$, the caret denoting omission. That is, the poly-contour $\Gamma$ is a product of $N$ contours, the $i$ 'th of which is V-shaped and connects the points $\left(\lambda^{k_{i}\left(\delta_{i}+1\right)} \infty, 0, \lambda^{k_{i} \delta_{i}} \infty\right)$ in the $x_{i}$ plane with two straight lines called 'spokes'; here $\lambda=e^{\frac{2 \pi_{i}}{d}}$, and $\delta_{i}=0,1, \ldots, a_{i}-2$; the $\delta_{i}=a_{i}-1$ contour is easily seen to be the sum (concatenation) of the others and is hence omitted as uninteresting. The choice of these contours ensures that each $x_{i}^{a_{i}}>0$, whence the integrals converge. It is amusing to note that this set of rather simple choices often suffices.

Expanding (2.12),

$$
\begin{gathered}
\varpi_{\left(\delta_{0}, \ldots, \widehat{\delta}_{m}, \ldots, \delta_{N}\right)}^{(m)}=C \sum_{n_{\beta}=0}^{\infty} \prod_{\beta} \frac{\phi_{\beta}^{n_{\beta}}}{n_{\beta} !} \int_{0}^{\infty} \mathrm{d} s s^{\|n\|} e^{-s} I_{\delta}(s) \\
I_{\delta}(s) \stackrel{\text { def }}{=} \prod_{i}^{\prime} \int_{V\left(\delta_{i}\right)} \mathrm{d} x_{i} e^{-s x_{i}^{a_{i}}} x_{i}^{q_{i} \cdot n}
\end{gathered}
$$

where $\int_{V\left(\delta_{i}\right)} \mathrm{d} x_{i}$ denotes the integral in the $x_{i}$-plane, along the $V$-shaped contour connecting the points $\left(\lambda^{k_{i}\left(\delta_{i}+1\right)} \infty, 0, \lambda^{k_{i} \delta_{i}} \infty\right)$, and

$$
\|n\| \stackrel{\text { def }}{=} \sum_{\alpha} n_{\alpha}, \quad q_{i} \cdot n \stackrel{\text { def }}{=} \sum_{\alpha} q_{i}^{\alpha} n_{\alpha} .
$$

Next, we use

$$
\int_{V\left(\delta_{i}\right)} \mathrm{d} \xi \xi^{\nu} e^{-s \xi^{a_{i}}}=\lambda^{k_{i} \delta_{i}(\nu+1)}\left(1-\lambda^{k_{i}(\nu+1)}\right) \frac{k_{i}}{d} \frac{\Gamma\left(\frac{k_{i}(\nu+1)}{d}\right)}{s^{k_{i}(\nu+1) / d}} .
$$

Therefore

$$
\begin{gathered}
\dot{\varpi}_{\left(\delta_{0}, \ldots, \widehat{\delta}_{m}, \ldots, \delta_{N}\right)}^{(m)}=\frac{C}{d^{N}} \sum_{n_{\beta}=0}^{\infty} \prod_{\beta} \frac{\phi_{\beta}^{n_{\beta}}}{n_{\beta} !}\left[\int_{0}^{\infty} \mathrm{d} s e^{-s}{ }_{s}^{\left[\frac{k_{m}}{d}\left(1+q_{i} \cdot n\right)-1\right]}\right] \\
\times \prod_{i}^{\prime} k_{i} \lambda^{k_{i} \delta_{i}\left(1+q_{i} \cdot n\right)}\left(1-\lambda^{k_{i}\left(1+q_{i} \cdot n\right)}\right) \Gamma\left(\frac{k_{i}}{d}\left(1+q_{i} \cdot n\right)\right) \\
-4-
\end{gathered}
$$


and we find that

$$
\begin{aligned}
\varpi_{\left(\delta_{0}, \ldots, \hat{\delta}_{m}, \ldots, \delta_{N}\right)}^{(m)}= & \frac{C \prod_{j} k_{j}}{k_{m} d^{N}}\left[\prod_{i}^{\prime} \lambda^{k_{i} \delta_{i}}\right] \sum_{\beta} \prod_{\beta} \frac{\left(\lambda^{\left(\Sigma_{j}^{\prime} \delta_{j} k_{j} q_{i}^{\beta}\right)} \phi_{\beta}\right)^{n_{\beta}}}{n_{\beta} !} \\
& \times \prod_{i} \Gamma\left(\frac{k_{i}}{d}\left(1+q_{i} \cdot n\right)\right) \prod_{j}^{\prime}\left(1-\lambda^{k_{j}\left(1+q_{i} \cdot n\right)}\right)
\end{aligned}
$$

There are $a_{i}-1$ choices of $\delta_{i}$ for each $i=0, \ldots, N, i \neq m$, yielding altogether $\Pi_{i}^{\prime}\left(a_{i}-1\right)$ possible poly-contours. This then is the upper limit on the number of distinct periods (2.18). In the worked examples, this turns out to be bigger than the total number of periods and, in fact, all $b_{3}=2\left(b_{2,1}+1\right)$ periods may be represented in this way. This construction of cycles from spokes is further detailed in the appendix.

Consider, on the other hand, the integral over the arc at infinity subtended between the two spokes of the above V-shaped contour. On the arc, take $x=r e^{i \theta}$ and let $\theta$ run from $\frac{2 \pi \delta}{a}$ to $\frac{2 \pi(6+1)}{a}$. Then

$$
I=\lim _{r \rightarrow \infty} \int \mathrm{d} x x^{\sigma} e^{-s x^{\alpha}}=\lim _{r \rightarrow \infty} i r^{\sigma+1} \int_{\frac{2 \pi \delta}{a}}^{\frac{2 \pi(\delta+1)}{a}} \mathrm{~d} \theta e^{i(\sigma+1) \theta-s r^{a} e^{i a \theta}} .
$$

Since $r^{a}$ is large, we expand in a power series, do the integral, and find a confluent hypergeometric function:

$$
\begin{aligned}
I & =\lim _{r \rightarrow \infty} \sum_{n=0}^{\infty} \frac{i(-s)^{n} r^{\sigma+1+a n}}{n !} \int_{\frac{2 \pi \delta}{a}}^{\frac{2 \pi(\delta+1)}{a}} \mathrm{~d} \theta e^{i \theta(\sigma+1+a n)} \\
& =e^{2 \pi i \delta \frac{\sigma+1}{a}}\left(e^{2 \pi i \frac{\sigma+1}{a}}-1\right) \lim _{r \rightarrow \infty} \sum_{n=0}^{\infty} \frac{(-s)^{n} r^{\sigma+1+a n}}{(\sigma+1+a n) n !} \\
& =e^{2 \pi i \delta \frac{\sigma+1}{a}}\left(e^{2 \pi i \frac{\sigma+1}{a}}-1\right) \lim _{r \rightarrow \infty} \sum_{n=0}^{\infty} \frac{r^{\sigma+1}}{a} \frac{\left(-s r^{a}\right)^{n}}{n !} \frac{\Gamma\left(n+\frac{\sigma+1}{a}\right)}{\Gamma\left(n+1+\frac{\sigma+1}{a}\right)} \\
& =e^{2 \pi i \delta \frac{\sigma+1}{a}}\left(e^{2 \pi i \frac{\sigma+1}{a}}-1\right) \lim _{r \rightarrow \infty} \frac{r^{\sigma+1}}{\sigma+1} F_{1}\left(\frac{\sigma+1}{a}, \frac{\sigma+1}{a}+1 ;-s r^{a}\right) .
\end{aligned}
$$

Now the limit from Ref. [20], p.278, noting that the argument is going to $-\infty$, finally yields

$$
\begin{aligned}
I & =e^{2 \pi i \delta \frac{\sigma+1}{a}}\left(e^{2 \pi i \frac{\sigma+1}{a}}-1\right) \lim _{r \rightarrow \infty} \frac{r^{\sigma+1}}{\sigma+1} \Gamma\left(\frac{\sigma+1}{a}+1\right)\left(s r^{a}\right)^{-\frac{\sigma+1}{a}} \\
& =\frac{1}{a} e^{2 \pi i \delta \frac{\sigma+1}{a}}\left(e^{2 \pi i \frac{\sigma+1}{a}}-1\right) \Gamma\left(\frac{\sigma+1}{a}\right) s^{-\frac{\sigma+1}{a}}
\end{aligned}
$$


which is exactly the same as the contribution from the V-shaped contour used above, except for a minus sign. Indeed, owing to the analyticity of the integrand in the wedge bounded by the $\mathrm{V}$-shaped contour and the arc at infinity, these two contours may be deformed into each other at will.

\subsection{Tadpole Models}

Following the polynomial classification methods of Arnold $[9,19,21,22]$, consider next the simplest of 'tadpole' type polynomials,

$$
P_{0}^{(\rho)}=x_{0}^{a_{0}}+x_{0} x_{1}^{a_{1}}
$$

In the course of calculating the periods one encounters integrals of the form

$$
\int_{\gamma_{0}} \mathrm{~d} x_{0} \int_{\gamma_{1}} \mathrm{~d} x_{1} e^{-s\left(x_{0}^{a_{0}}+x_{0} x_{1}^{a_{1}}\right)} x_{0}^{\sigma_{0}} x_{1}^{\sigma_{1}}
$$

wherein we must choose the contour $\gamma_{0} \times \gamma_{1}$ to ensure that the tadpole has positive real part. We will do this by requiring each term to have a positive real part. To ensure this for the 'Fermat' term, $x_{0}^{a_{0}}$, we restrict $x_{0}$ to be on the spokes

$$
x_{0}=\xi_{0} e^{2 \pi i \frac{r}{a_{0}}}, \quad r \in \mathbb{Z}, \quad 0 \leq \xi_{0}<\infty .
$$

We can then choose a contour $\gamma_{0}$ where $x_{0}$ follows $x_{0}=\xi_{0} e^{2 \pi i \frac{\delta_{0}+1}{a_{0}}}$ from $\infty$ to 0 , and then $x_{0}=\xi_{0} e^{2 \pi i \frac{\delta_{0}}{a_{0}}}$ from 0 to $\infty$. Label the contour by $\delta_{0}$, for which the values $0, \ldots, a_{0}-2$ describe independent paths.

To ensure that the term $x_{0} x_{1}^{a_{1}}$ has a positive real part, we require that

$$
\arg \left(x_{1}^{a_{1}}\right)=-2 \pi \frac{\left(\delta_{0}+\frac{1}{2}\right)}{a_{0}} .
$$

This means that the argument of $x_{0} x_{1}^{a_{1}}$ will be either $-\frac{\pi}{a_{0}}$ or $\frac{\pi}{a_{0}}$, and so as long as $a_{0}>2$, this has a positive real part. In the special case when $a_{0}=2$, we may change variables to a Fermat-type polynomial (see below). This means that $x_{1}$ lies on spokes defined by

$$
x_{1}=\xi_{1} e^{2 \pi i\left(\frac{s}{a_{1}}-\frac{\delta_{0}+\frac{1}{2}}{a_{1} a_{0}}\right)}, \quad s \in \mathbb{Z}, \quad 0 \leq \xi_{1}<\infty
$$

Taking adjacent spokes, we let $x_{1}$ follow the spoke with $s=\delta_{1}+1$ from $\infty$ to 0 , and the spoke $s=\delta_{1}$ from 0 to $\infty$, and we may choose $\delta_{1}$ from $0, \ldots, a_{1}-2$. 
We may now do the integral (2.23)

$$
\begin{aligned}
& I=\int_{\delta_{0} \times \delta_{1}} \mathrm{~d} x_{0} \mathrm{~d} x_{1} e^{-s\left(x_{0}^{a_{0}}+x_{0} x_{1}^{a_{1}}\right)} x_{0}^{\sigma_{0}} x_{1}^{\sigma_{1}} \\
& =\frac{1}{a_{0} a_{1}} e^{2 \pi i\left(\delta_{1}\left(\frac{\sigma_{1}+1}{a_{1}}\right)+\delta_{0}\left(\frac{\sigma_{0}+1}{a_{0}}-\frac{\sigma_{1}+1}{a_{1} a_{0}}\right)\right)}\left[1-e^{2 \pi i\left(\frac{\sigma_{1}+1}{a_{1}}\right)}\right]\left[1-e^{2 \pi i\left(\frac{\sigma_{0}+1}{a_{0}}-\frac{\sigma_{1}+1}{a_{1} a_{0}}\right)}\right] \\
& \quad \times \Gamma\left(\frac{\sigma_{1}+1}{a_{1}}\right) \Gamma\left(\frac{\sigma_{0}+1}{a_{0}}-\frac{\sigma_{1}+1}{a_{1} a_{0}}\right) s^{-\left(\frac{\sigma_{0}+1}{a_{0}}+\frac{\left(\sigma_{1}+1\right)\left(a_{0}-1\right)}{a_{1} a_{0}}\right)} .
\end{aligned}
$$

The extension to longer tadpole polynomials is straightforward. If the polynomial contains the third term, $x_{1} x_{2}^{a_{2}}$, then we insist that $x_{2}$ lie along the spokes

$$
x_{2}=\xi_{2} e^{2 \pi i\left(\frac{t}{a_{2}}-\frac{\delta_{1}+\frac{1}{2}}{a_{2} a_{1}}+\frac{\delta_{0}+\frac{1}{2}}{a_{2} a_{1} a_{0}}\right)}, \quad t \in \mathbb{Z}, \quad 0 \leq \xi_{2}<\infty .
$$

For adjacent spokes, take $t=\delta_{2}+1$ on the spoke towards the origin and $t=\delta_{2}$ on the way out. The corresponding integral is then obtained along the lines of (2.27). The diligent Reader will have no problem in iterating this procedure to obtain the integrals analogous to $(2.27)$.

\subsection{Loop Models}

Next, consider the simplest loop-type polynomial:

$$
P_{0}^{(O)}=x_{0}^{a_{0}} x_{1}+x_{0} x_{1}^{a_{1}}
$$

We assume that $x_{0}$ and $x_{1}$ will follow spokes so that for each $j=0,1$,

$$
\arg x_{j}^{a_{j}}=2 \pi \alpha_{j} \quad \text { implying } \quad x_{j}=\xi_{j} e^{2 \pi i \frac{\alpha_{j}+m}{a_{j}}}
$$

Choosing adjacent spokes for each $\left(x_{0}\right.$ comes in from $\infty$ on $\delta_{0}+1$, out on $\delta_{0}, x_{1}$ comes in from $\infty$ on $\delta_{1}+1$, out on $\delta_{1}$ ) means that the terms of the polynomial lie along the spokes

$$
\arg \left(x_{j}^{a_{j}} x_{1-j}\right)=2 \pi\left(\alpha_{j}+\frac{\alpha_{1-j}+\delta_{1-j}+1}{a_{1-j}}\right) \quad \text { or } \quad 2 \pi\left(\alpha_{j}+\frac{\alpha_{1-j}+\delta_{1-j}}{a_{1-j}}\right)
$$

We centre these on the positive real axis by solving the equations

$$
\alpha_{j}+\frac{\alpha_{1-j}+\delta_{1-j}+\frac{1}{2}}{a_{1-j}}=0
$$

which has the solutions

$$
\begin{gathered}
\alpha_{j}=\frac{\delta_{j}+\frac{1}{2}-a_{j}\left(\delta_{1-j}+\frac{1}{2}\right)}{a_{0} a_{1}-1} \\
-7-
\end{gathered}
$$


The integral now becomes

$$
\begin{aligned}
& I= \int_{\delta_{0} \times \delta_{1}} \mathrm{~d} x_{0} \mathrm{~d} x_{1} e^{-s\left(x_{0}^{a_{0}} x_{1}+x_{0} x_{1}^{a_{1}}\right)} x_{0}^{\sigma_{0}} x_{1}^{\sigma_{1}} \\
&=\frac{1}{a_{0} a_{1}-1} e^{2 \pi i\left(\frac{\left(\sigma_{0}+1\right)\left(\delta_{0} a_{1}-\delta_{1}\right)+\left(\sigma_{1}+1\right)\left(\sigma_{1} a_{0}-\delta_{0}\right)}{a_{0} a_{1}-1}\right)} s^{-\left(\frac{\left(\sigma_{0}+1\right)\left(a_{1}-1\right)+\left(\sigma_{1}+1\right)\left(a_{0}-1\right)}{a_{0} a_{1}-1}\right)} \\
& \quad \times\left[1-e^{2 \pi i\left(\frac{\left(\sigma_{0}+1\right) a_{1}-\left(\sigma_{1}+1\right)}{a_{0} a_{1}-1}\right)}\right]\left[1-e^{2 \pi i\left(\frac{\left(\sigma_{1}+1\right) a_{0}-\left(\sigma_{0}+1\right)}{a_{0} a_{1}-1}\right)}\right] \\
& \quad \times \Gamma\left(\frac{\left(\sigma_{0}+1\right) a_{1}-\left(\sigma_{1}+1\right)}{a_{0} a_{1}-1}\right) \Gamma\left(\frac{\left(\sigma_{1}+1\right) a_{0}-\left(\sigma_{0}+1\right)}{a_{0} a_{1}-1}\right)
\end{aligned}
$$

For longer loops with $n$ fields in the loop, the corresponding system of $n$ equations for the $n \alpha_{i}$ 's is solved as easily and the integral (2.34) generalizes straightforwardly.

\subsection{On Non-Invertible Models}

The above simple models may be used as 'building blocks' for Calabi-Yau or LandauGinzburg orbifold models where the defining polynomial (superpotential) is transverse and has as many terms as there are coordinates. This by no means exhausts all the possibilities; the recently compiled complete lists of non-degenerate $(2,2)$ Landau-Ginzburg vacua with $c=9[22,23,24]$ include 'non-invertible' models. These are non-degenerate only upon including more terms than there are coordinates. In addition, we may want to consider more general theories, regardless of stringy application. Thus, rather than trying to discuss all possible polynomials, we give one such non-invertible example and hope that generalizations will be equally clear.

Similar to tadpoles are polynomials of the form

$$
P_{0}^{(R)}=x_{0}^{a_{0}} x_{1}+x_{1}^{a_{1}}+x_{1} x_{2}^{a_{2}},
$$

where, for homogeneity, $a_{0}=\left(d-k_{1}\right) / k_{0}$ and $a_{2}=\left(d-k_{1}\right) / k_{2}$. The method of choosing contours is similar. The middle term has a positive real part as long as $x_{1}$ is on the spokes $x_{1}=\xi_{1} e^{2 \pi i \frac{r}{a_{1}}}$, and so in a now-familiar way we choose adjacent spokes $r=\delta_{1}+1$ and $n=\delta_{1}$ to form the $\mathrm{V}$-shaped contour which is equivalent to the arc at infinity. The positivity requirements for the first and third terms lead us to

$$
\arg \left(x_{0}^{a_{0}}\right)=\arg \left(x_{2}^{a_{2}}\right)=-\frac{2 \pi\left(\delta_{1}+\frac{1}{2}\right)}{a_{1}},
$$

so we restrict $x_{0}$ and $x_{2}$ to be on the spokes

$$
\begin{array}{ll}
x_{0}=\xi_{0} e^{2 \pi i\left(\frac{s}{a_{0}}-\frac{\delta_{1}+\frac{1}{2}}{a_{1} a_{0}}\right)} & s \in \mathbb{Z}, \quad 0 \leq \xi_{0}<\infty, \\
x_{2}=\xi_{2} e^{2 \pi i\left(\frac{t}{a_{2}}-\frac{\delta_{1}+\frac{1}{2}}{a_{1} a_{2}}\right)} & s \in \mathbb{Z}, \quad 0 \leq \xi_{2}<\infty,
\end{array}
$$


and form contours with $s_{\mathrm{in}}=\delta_{0}+1$ and $s_{\mathrm{out}}=\delta_{0}$, and $t_{\mathrm{in}}=\delta_{2}+1$ and $t_{\mathrm{out}}=\delta_{2}$.

Note, however, that the polynomial (2.35) cannot be used to construct a LandauGinzburg orbifold, as it is degenerate: $x_{0}=x_{2}=0$ and $x_{1}$ arbitrary parametrize a 'flat direction', where both $P_{0}^{(R)}$ and $\mathrm{d} P_{0}^{(R)}$ vanish. This is remedied [21] by deforming $P_{0}^{(R)}$ into

$$
\widetilde{P}_{\varepsilon}^{(R)}=P_{0}^{(R)}+\varepsilon x_{0}{ }^{p} x_{2}^{q},
$$

where, for homogeneity, $p$ and $q$ are related by $p k_{0}+q k_{2}=a_{1} k_{1}$. It is easy to check that, for nonzero and however small $\varepsilon$, the deformed polynomial (2.38) is non-degenerate. The positivity condition in (2.6) then implies a restriction on the possible values of $s, t$ :

$$
k_{1}\left(\delta_{1}+\frac{1}{2}\right)-\frac{d-k_{1}}{4}<p k_{0} s+q k_{2} t<k_{1}\left(\delta_{1}+\frac{1}{2}\right)+\frac{d-k_{1}}{4}
$$

which may be possible to neglect for sufficiently small $\varepsilon$. In any case, treating $\varepsilon$ as another parameter $\phi_{\alpha}$, we calculate the period as described above. The limit $\varepsilon \rightarrow 0$ is then taken to provide the value of the period for the otherwise degenerate Landau-Ginzburg orbifold (2.35).

\subsection{On Fermatization}

Alternatively, the period integrals may always be calculated by changing variables and transforming any given model into (an orbifold of) a 'Fermat' model. In addition, contours for the original model may be obtained from those in the 'fermatization' using the inverse transformation. In practice this approach may be preferrable, especially in the cases when part of the polynomial is of the non-invertible type discussed in the previous subsection.

As in Ref. [25], consider 'fermatizing' the loop

$$
x_{1}^{a_{1}} x_{2}+x_{1} x_{2}^{a_{2}} \rightarrow y_{1}^{b_{1}}+y_{2}^{b_{2}}
$$

For the Jacobian to be constant we need

$$
b_{1}=\frac{a_{1} a_{2}-1}{a_{2}-1}, \quad b_{2}=\frac{a_{1} a_{2}-1}{a_{1}-1},
$$

and hence the coordinate transformation is

$$
\begin{array}{cc}
y_{1}=x_{1}^{\frac{a_{1}\left(a_{2}-1\right)}{a_{1} a_{2}-1}} x_{2}^{\frac{a_{2}-1}{a_{1} a_{2}-1}} \quad x_{1}=y_{1}^{\frac{a_{2}}{a_{2}-1}} y_{2}^{\frac{-1}{a_{1}-1}} \\
y_{2}=x_{1}^{\frac{a_{1}-1}{a_{1} a_{2}-1}} x_{2}^{\frac{a_{2}\left(a_{1}-1\right)}{a_{1} a_{2}-1}} \quad x_{2}=y_{1}^{\frac{1}{a_{2}-1}} y_{2}^{\frac{a_{1}}{a_{1}-1}} \\
-9-
\end{array}
$$


The type of integral that comes up in period calculations then becomes

$$
\begin{aligned}
& \int \mathrm{d}^{2} x x_{1}^{\sigma_{1}} x_{2}^{\sigma_{2}} e^{-s\left(x_{1}^{a_{1}} x_{2}+x_{1} x_{2}^{a_{2}}\right)}= \\
& \quad \frac{a_{1} a_{2}-1}{\left(a_{1}-1\right)\left(a_{2}-1\right)} \int \mathrm{d}^{2} y y_{1}^{\frac{\sigma_{1} a_{2}-\sigma_{2}}{a_{2}-1}} y_{2}^{\frac{\sigma_{2} a_{1}-\sigma_{1}}{a_{1}-1}} e^{-s\left(y_{1}^{\frac{a_{1} a_{2}-1}{a_{2}-1}}+y_{2}^{\frac{a_{1} a_{2}-1}{a_{1}-1}}\right)}
\end{aligned}
$$

Calculating the second double integral (2.43) in the manner of $\S 2.1$ gives the same answer as calculating the original double integral (2.34) in the manner of $\$ 2.3$.

One may worry that the fractional exponents $b_{i}$ may be smaller than 2 and hence our arguments used for the Fermat models of $\S 2.1$ would not be relevant. However, this is not the case. To see this in the situation described above, rewrite Eqs. (2.41) as

$$
b_{1}=\left(\frac{a_{1}-1}{a_{2}-1}\right)+a_{1}, \quad b_{2}=\left(\frac{a_{2}-1}{a_{1}-1}\right)+a_{2}, \quad a_{1}, a_{2} \in \mathbb{Z} .
$$

Noting that $a_{i} \geq 2$ we find that this is the case for the $b_{i}$ as well. Thus our results from $\S 2.1$ still holds. This argument can be seen to apply for any other 'fermatization' as well. Finally, if $a_{1} \neq a_{2}$, at least one of $b_{i}$ is non-integral. While this does not hinder the evaluation of the period integral, a Fermat model with integral powers is easily obtained through a further change of variables, compensated by passing to a suitable quotient.

\subsection{Landau-Ginzburg Vacua}

In the previous section we discussed a rather large class of Calabi-Yau models, not necessarily of dimension three, for which we can write down a set of periods in a manifest homology basis. It is, however, clear that we do not need to restrict to theories for which a nonlinear Calabi-Yau $\sigma$-model interpretation is known. Such examples are found among Landau-Ginzburg string vacua with more than five superfields; for a recent classification of such theories see [24].

Let us consider the usual action of an $N=2$ superconformal Landau-Ginzburg theory

$$
\int d^{2} z d^{2} \theta d^{2} \bar{\theta} K\left(X_{i}, \bar{X}_{i}\right)+\left(\int d^{2} z d^{2} \theta P\left(X_{i}\right)+c . c\right) .
$$

where $K$ is the Kähler potential and $P$, the superpotential, is a holomorphic function of the $N=2$ chiral superfields $X_{i}\left(z, \bar{z}, \theta^{+}, \theta^{-}\right)$. Due to nonrenormalization theorems, $P$ is not renormalized (up to scaling) and hence will characterize the theory (modulo irrelevant perturbations coming from the Kähler potential [26].) Let $P$ be a polynomial in the superfields $X_{i}, i=0, \ldots, n$, and, moreover, let it be quasi-homogeneous of degree $d$, i.e., under rescaling of the world-sheet

$$
X_{i} \mapsto \lambda^{k_{i}} X_{i}, \quad P\left(X_{i}\right) \mapsto \lambda^{d} P\left(X_{i}\right)
$$


The central charge is straightforward to compute [26], and is given by $c=6 \sum_{i=0}^{n}\left(\frac{1}{2}-q_{i}\right)$ with $q_{i}=k_{i} / d$ the charge under the left-moving $U(1)$-current $J_{0}$. In order to ensure $(2,2)$ world-sheet supersymmetry we need to consider the Landau-Ginzburg orbifold $P\left(X_{i}\right) / j$ where $j=e^{2 \pi i J_{0}}$. This projects onto the integer charged states and preserves the supersymmetry. We will not restrict our attention to models with $c=9$. Rather, set $\hat{c}=c / 3$ and the expression for the central charge can rewritten as

$$
\sum_{i=0}^{n} k_{i}=k d
$$

where $k=(n+1-\hat{c}) / 2$.

For our purposes it is sufficient to note the isomorphism between the chiral ring and the ring of polynomials modulo the ideal generated by $\partial P\left(x_{i}\right)$; the $x_{i}$ are the lowest order componets of the chiral superfields $X_{i}$ in the point field limit.

Following our discussion in section 2 the relevant object to consider for the periods is given by

$$
\omega_{j}\left(\phi_{\alpha}\right)=C \int_{\Gamma_{j}} \frac{\prod_{i=0}^{n} \mathrm{~d} x_{i}}{\left(P_{\phi}\right)^{k}} ;
$$

for $n+1-\hat{c}=2$ this reproduces (2.5). In particular $n=4, \hat{c}=3$ we get the wellknown expression for the period of a Calabi-Yau three-fold. Note that because of the scale invariance the integrand only depends on $n$ of the $n+1$ coordinates. Thus, just as for the Calabi-Yau models we can choose to set one of the $x_{i}$ to one and we obtain the equivalent of (2.5). The polynomial is then expanded about the $\phi_{\alpha}$ by splitting $P_{\phi}$ into a reference polynomial $P_{0}$ and a perturbation $\Delta$; thus,

$$
\begin{aligned}
\frac{1}{P_{\phi}^{k}} & =\frac{1}{\left(P_{0}-\Delta\right)^{k}}=\sum_{n=0}^{\infty} \frac{(n+k-1) !}{n !} \frac{\Delta^{n}}{P_{0}^{n+k}} \\
& =\sum_{n=0}^{\infty} \frac{\Delta^{n}}{n !} \int_{0}^{\infty} d s s^{n+k-1} e^{-s P_{0}}, \quad \Re e\left(P_{0}\right)>0
\end{aligned}
$$

The discussion for the various types of polynomials then applies verbatim and we refer the reader to the following section for an example and explicit calculation.

\section{Examples}

Having found suitable contours for the building blocks of reference polynomials, we may apply our results to finding periods for any of the types listed in [8]. Further, this method adapts well to many (weighted) complete intersection spaces $[1,14,15,16,17]$, generalized Calabi-Yau manifolds [12], and Landau-Ginzburg vacua [22,23,24]. 


\subsection{A Known Example}

We start with a 2-parameter family of hypersurfaces $\mathbb{P}_{(3,2,2,7,7)}^{4}[21]_{-78}^{50,11}$ from Ref. [11]:

$$
P_{\phi_{0}, \phi_{1}}=x_{0}^{7}+x_{1}^{7} x_{3}+x_{3}^{3}+x_{2}^{7} x_{4}+x_{4}^{3}-\phi_{0} x_{0} x_{1} x_{2} x_{3} x_{4}+\phi_{1} x_{0}^{3} x_{1}^{3} x_{2}^{3}
$$

the superscripts 50,11 indicate the Hodge numbers $b_{2,1}, b_{1,1}$ and the subscript +6 is the Euler characteristic $\chi_{E}=2\left(b_{1,1}-b_{2,1}\right)$. We will calculate the periods in the coordinate patch where $x_{0}=1$, and use the general results of $\S 2.2$.

$$
\begin{aligned}
\varpi_{\left(\delta_{1}, \delta_{2}, \delta_{3}, \delta_{4}\right)=} & C \int_{\left(\delta_{1}, \delta_{2}, \delta_{3}, \delta_{4}\right)} \mathrm{d}^{4} x \int_{0}^{\infty} \mathrm{d} s e^{-\left.s P\right|_{x_{0}=1}} \\
= & C \sum_{n=0}^{\infty} \sum_{m=0}^{\infty} \frac{\phi_{0}^{n}}{n !} \frac{\left(-\phi_{1}\right)^{m}}{m !} \int_{0}^{\infty} \mathrm{d} s e^{-s} s^{n+m} \\
& \times \int_{\left(\delta_{1}, \delta_{2}, \delta_{3}, \delta_{4}\right)} \mathrm{d}^{4} x e^{-s\left(x_{1}^{7} x_{3}+x_{3}^{3}+x_{2}^{7} x_{4}+x_{4}^{3}\right)} x_{1}^{n+3 m} x_{2}^{n+3 m} x_{3}^{n} x_{4}^{n} \\
=\frac{C}{3^{2} 7^{2}} & \sum_{n=0}^{\infty} \sum_{m=0}^{\infty} \frac{\phi_{0}^{n}}{n !} \frac{\left(-\phi_{1}\right)^{m}}{m !} e^{2 \pi i\left(\left(\delta_{1}+\delta_{2}\right)\left(\frac{n+3 m+1}{7}\right)+\left(\delta_{3}+\delta_{4}\right)\left(\frac{2 n-m+2}{7}\right)\right)} \\
& \times\left(1-e^{2 \pi i\left(\frac{n+3 m+1}{7}\right)}\right)^{2}\left(1-e^{2 \pi i\left(\frac{2 n-m+2}{7}\right)}\right)^{2} \\
& \times \Gamma^{3}\left(\frac{n+3 m+1}{7}\right) \Gamma^{2}\left(\frac{2 n-m+2}{7}\right)
\end{aligned}
$$

where the range of the $\delta_{i}$ are $0 \leq \delta_{1}, \delta_{2} \leq 5,0 \leq \delta_{3}, \delta_{4} \leq 1$. This would lead to a set of $2^{2} 6^{2}=144$ possible contours, but only six are in fact independent. This is easily seen as follows. For the deformations considered, the period dependence on $\delta_{1}$ is the same as that on $\delta_{2}$, and the dependence on $\delta_{3}$ and $\delta_{4}$ is just twice that of the dependence on $\delta_{1}$. That is, letting $\delta_{3} \rightarrow \delta_{3}+1$ is the same as letting $\delta_{1} \rightarrow \delta_{1}+2$. Knowing this, we may choose $\delta_{2}=\delta_{3}=\delta_{4}=0$.

Rearranging the expression for the periods by using

$$
\left(1-e^{2 \pi i \alpha}\right) \Gamma(\alpha)=-2 \pi i \frac{e^{\pi i \alpha}}{\Gamma(1-\alpha)},
$$

and writing $l=n+1$, we arrive at the more compact expression for the periods

$$
\varpi_{\left(\delta_{1}\right)}^{(0)}=\frac{(2 \pi)^{4} C}{3^{2} 7^{2} \phi_{0}} \sum_{l=1}^{\infty} \sum_{m=0}^{\infty} \frac{e^{2 \pi i l\left(\frac{\delta_{1}+3}{7}\right)} \phi_{0}^{l}}{\Gamma(l)} \frac{e^{\pi i m\left(\frac{6 \delta_{1}-3}{7}\right)} \phi_{1}^{m}}{m !} \frac{\Gamma\left(\frac{l+3 m}{7}\right)}{\Gamma^{2}\left(1-\frac{l+3 m}{7}\right) \Gamma^{2}\left(1-\frac{2 l-m}{7}\right)} .
$$

For $\delta_{1}=0$, this is the period calculated in Ref. [11]. 
The same example, when calculated in the patch $x_{3}=1$, yields almost the same periods. After all the rearrangements and upon realizing that the different periods can all be obtained by varying only $\delta_{0}$, we get

$$
\varpi_{\left(\delta_{0}\right)}^{(3)}=\frac{(2 \pi)^{4} C}{3^{2} 7^{2} \phi_{0}} \sum_{l=1}^{\infty} \sum_{m=0}^{\infty} \frac{e^{2 \pi i l\left(\frac{\delta_{0}+3}{7}\right)} \phi_{0}^{l} e^{\pi i m\left(\frac{6 \delta_{0}-3}{7}\right)} \phi_{1}^{m}}{\left(1+e^{2 \pi i\left(\frac{1+3 m}{7}\right)}\right) \Gamma(l) m !} \frac{\Gamma\left(\frac{l+3 m}{7}\right)}{\Gamma^{2}\left(1-\frac{l+3 m}{7}\right) \Gamma^{2}\left(1-\frac{2 l-m}{7}\right)} .
$$

We find that

$$
\varpi_{\left(\delta_{0}\right)}^{(3)}+\varpi_{\left(\delta_{0}+1\right)}^{(3)}=\varpi_{\left(\delta_{1}\right)}^{(0)}
$$

that is, we recover the expression in $x_{0}=1$ coordinate patch. So the periods in different patches are just linear combinations of each other.

Most notably, the action of (a subgroup of) the modular group found in Ref. [11], can be realized in the present analysis simply by changing the choice of the poly-contour:

$$
\left(\phi_{0}, \phi_{1}\right) \longrightarrow\left(\lambda \phi_{0}, \lambda^{3} \phi_{1}\right) \cong \delta_{1} \longrightarrow \delta_{1}+1
$$

The erudite Reader will be reminded of Dehn twists, which in the case of Riemann surfaces generate the modular group; however, for the case of Calabi-Yau 3-spaces, the general theory of moduli space and modular group is far from that well developed and we do not know how much of the modular group can be uncovered in this simple fashion. We hope to return to a more detailed analysis of this relation between these simple operations on the contours and periods, and the elements of the modular group.

\subsection{A Mixed Example}

Consider the somewhat peculiar example found as the penultimate item in Table 2 of Ref. [9]. It concerns the family of models $\mathbb{P}_{(2,17,17,6,9)}[51]_{+6}^{31,34}$ and their mirror models, $\left\{\mathbb{P}_{(3,16,17,6,9)}[51] / \mathbb{Z}_{2}:(0,0,0,1,1)\right\}_{-6}^{34,31}$. The reference defining polynomials (superpotentials) are

$$
\begin{aligned}
& P_{0}=\left[x_{0}{ }^{17} x_{1}+x_{1}{ }^{3}\right]+x_{2}{ }^{3}+\left[x_{3}{ }^{7} x_{4}+x_{3} x_{4}{ }^{5}\right] \\
& \widehat{P}_{0}=\left[y_{0}{ }^{17}+y_{0} y_{1}{ }^{3}\right]+y_{2}{ }^{3}+\left[y_{3}{ }^{7} y_{4}+y_{3} y_{4}{ }^{5}\right] .
\end{aligned}
$$

The square brackets merely group the irreducible models: a 'tadpole', a 'Fermat' and a 'loop' term; $\widehat{P}_{0}$ was obtained by 'transposition' [9].

Suppose we add the fundamental deformation to $P_{0}$, so that $P=P_{0}-\phi_{0} x_{0} x_{1} x_{2} x_{3} x_{4}$. Then the periods in the coordinate patch where $x_{2}=1$ become

$$
\begin{aligned}
\varpi^{(2)}=\frac{C}{6} & \cdot 17^{2} \sum_{m=0}^{\infty} e^{2 \pi i(m+1)\left(\frac{1}{17} \delta_{0}+\frac{16}{51} \delta_{1}+\frac{2}{17} \delta_{3}+\frac{3}{17} \delta_{4}\right)} \frac{\phi_{0}^{m}}{m !} \\
& \times\left(1-e^{2 \pi i\left(\frac{m+1}{17}\right)}\right)\left(1-e^{2 \pi i\left(\frac{16(m+1)}{51}\right)}\right)\left(1-e^{2 \pi i\left(\frac{2(m+1)}{17}\right)}\right)\left(1-e^{2 \pi i\left(\frac{3(m+1)}{17}\right)}\right) \\
& \times \Gamma\left(\frac{m+1}{17}\right) \Gamma\left(\frac{16(m+1)}{51}\right) \Gamma\left(\frac{2(m+1)}{17}\right) \Gamma\left(\frac{3(m+1)}{17}\right) \Gamma\left(\frac{m+1}{3}\right)
\end{aligned}
$$


We see that the dependence on different contours can be summed up by varying only $\delta_{1}$, and so, with a slight change of notation, we find

$$
\begin{aligned}
& \varpi_{\delta}^{(2)}=\frac{C}{6} 17^{2} \\
& \sum_{m=0}^{\infty} e^{2 \pi i \delta\left(\frac{(m+1)}{\delta 1}\right)} \frac{\phi_{0}^{m}}{m !} \\
& \times\left(1-e^{2 \pi i\left(\frac{m+1}{17}\right)}\right)\left(1-e^{2 \pi i\left(\frac{18(m+1)}{51}\right)}\right)\left(1-e^{2 \pi i\left(\frac{2(m+1)}{17}\right)}\right)\left(1-e^{2 \pi i\left(\frac{3(m+1)}{17}\right)}\right) \\
& \quad \times \Gamma\left(\frac{m+1}{17}\right) \Gamma\left(\frac{16(m+1)}{51}\right) \Gamma\left(\frac{2(m+1)}{17}\right) \Gamma\left(\frac{3(m+1)}{17}\right) \Gamma\left(\frac{m+1}{3}\right) .
\end{aligned}
$$

Now if we add another deformation so that $P=P_{0}-\phi_{0} x_{0} x_{1} x_{2} x_{3} x_{4}-\phi_{1} x_{0}^{17} x_{2}$, we discover that the periods are

$$
\begin{aligned}
\varpi^{(2)} & =\frac{C}{6 \cdot 17^{2}} \sum_{m=0}^{\infty} \sum_{n=0}^{\infty} e^{2 \pi i\left(\frac{m+1}{17} \delta_{0}+\frac{16 m-17 n+16}{51} \delta_{1}+\frac{2(m+1)}{17} \delta_{3}+\frac{3(m+1)}{17} \delta_{4}\right)} \frac{\phi_{0}^{m}}{m !} \frac{\phi_{1}^{n}}{n !} \\
& \times\left(1-e^{2 \pi i\left(\frac{m+1}{17}\right)}\right)\left(1-e^{2 \pi i\left(\frac{16 m-17 n+16}{51}\right)}\right)\left(1-e^{2 \pi i\left(\frac{2(m+1)}{17}\right)}\right)\left(1-e^{2 \pi i\left(\frac{3(m+1)}{17}\right)}\right) \\
& \times \Gamma\left(\frac{m+17 n+1}{17}\right) \Gamma\left(\frac{16 m-17 n+16}{51}\right) \Gamma\left(\frac{2(m+1)}{17}\right) \Gamma\left(\frac{3(m+1)}{17}\right) \\
& \times \Gamma\left(\frac{m+n+1}{3}\right) .
\end{aligned}
$$

We need only vary $\delta_{0}$ and $\delta_{1}$ to find all the periods.

Adding one more deformation, the polynomial becomes $P=P_{0}-\phi_{0} x_{0} x_{1} x_{2} x_{3} x_{4}-$ $\phi_{1} x_{0}^{17} x_{2}-\phi_{2} x_{3}^{4} x_{4}^{3}$. The periods are then easily seen to be:

$$
\begin{aligned}
\varpi^{(2)} & =\frac{C}{6 \cdot 17^{2}} \sum_{m=0}^{\infty} \sum_{n=0}^{\infty} \sum_{p=0}^{\infty} e^{2 \pi i\left(\frac{m+1}{17} \delta_{0}+\frac{16 m-17 n+16}{51} \delta_{1}+\frac{4 m+17 p+4}{34} \delta_{3}+\frac{6 m+17 p+6}{34} \delta_{4}\right)} \frac{\phi_{0}^{m}}{m !} \frac{\phi_{1}^{n}}{n !} \frac{\phi_{2}^{p}}{p !} \\
& \times\left(1-e^{2 \pi i\left(\frac{m+1}{17}\right)}\right)\left(1-e^{2 \pi i\left(\frac{16 m-17 n+16}{51}\right)}\right)\left(1-e^{2 \pi i\left(\frac{4 m+17 p+4}{34}\right)}\right)\left(1-e^{2 \pi i\left(\frac{6 m+17 p+6}{34}\right)}\right) \\
& \times \Gamma\left(\frac{m+17 n+1}{17}\right) \Gamma\left(\frac{16 m-17 n+16}{51}\right) \Gamma\left(\frac{4 m+17 p+4}{34}\right) \Gamma\left(\frac{6 m+17 p+6}{34}\right) \\
& \times \Gamma\left(\frac{m+n+1}{3}\right) .
\end{aligned}
$$

Judicious use of Eq. (3.3) allows the periods to be written in a more compact form; we display this series of sets of periods in this fashion to show the ease of application of our formalism. The Reader will see that adding further perturbations presents no barrier to the calculation of periods. 


\subsection{A Twisted Example}

The first example in Ref. [25] concerns a pair of manifolds related by a fractional transformation

$$
\begin{array}{lll}
\mathcal{M}: & \mathbb{P}_{(1,7,2,2,2)}[14]_{-240}^{122,2} & P_{0}=x_{1}^{14}+x_{2}^{2}+x_{3}^{7}+x_{4}^{7}+x_{5}^{7} \\
\tilde{\mathcal{M}}: & \mathbb{P}_{(1,3,1,1,1)}[7]_{-240}^{122,2} & \tilde{P}_{0}=y_{1}^{7}+y_{1} y_{2}^{2}+y_{3}^{7}+y_{4}^{7}+y_{5}^{7} .
\end{array}
$$

All $122 b_{21}$ states of $\tilde{\mathcal{M}}$ are represented by deformations of the polynomial $\tilde{P}_{0}$, and are untwisted states in the corresponding Landau-Ginzburg model. From the fractional transformation

$$
\begin{array}{ll}
y_{1}=x_{1}^{2} & x_{1}=y_{1}^{\frac{1}{2}} \\
y_{2}=x_{2} / x_{1} & x_{2}=y_{1}^{\frac{1}{2}} y_{2}
\end{array}
$$

we see that there are 15 deformations

$$
\left\{y_{2}\right\} \otimes\left\{y_{3}^{a_{3}} y_{4}^{a_{4}} y_{5}^{a_{5}} \mid 0 \leq a_{i} \leq 4, a_{3}+a_{4}+a_{5}=4\right\}
$$

which get mapped to rational functions

$$
\left\{\frac{x_{2}}{x_{1}}\right\} \otimes\left\{x_{3}^{a_{3}} x_{4}^{a_{4}} x_{5}^{a_{5}} \mid 0 \leq a_{i} \leq 4, a_{3}+a_{4}+a_{5}=4\right\} .
$$

Analyzing the Landau-Ginzburg model described by $P_{0}$ we find 107 untwisted and 15 twisted states, the former images under (3.14) of deformations of $\tilde{P}_{0}$ and the latter of the form

$$
\left\{\mid\left(\frac{3}{7}, \frac{3}{7}\right)_{(c, c)}^{7}\right\} \otimes\left\{x_{3}^{a_{3}} x_{4}^{a_{4}} x_{5}^{a_{5}} \mid 0 \leq a_{i} \leq 4, a_{3}+a_{4}+a_{5}=4\right\} .
$$

Thus we conclude that there is a correspondence between the twisted vacuum and a rational function, $\frac{x_{2}}{x_{1}} \leftrightarrow \mid\left(\frac{3}{7}, \frac{3}{7}\right\rangle_{(c, c)}^{7}$. Note that there is no deformation of $P_{0}$ that is not mapped to a deformation of $\tilde{P}_{0}$. One might have thought from (3.14) that some square roots of $y_{1}$ might pop up, but a simple analysis shows that they cannot. To make a deformation of $P_{0}$ of charge 1 , every odd power of $x_{1}$ must be accompanied by an odd power of $x_{2}$, and vice versa, and hence the image under (3.14) has an integral power of $y_{1}$. This is related to the fact that the $\mathbb{Z}_{2}$ identification necessary to make (3.14) a one-one map is part of the projective equivalence identification on $\mathcal{M}$. The $\mathbb{Z}_{2}$ acts on the $x$ 's as

$$
\left(x_{1}, x_{2}, x_{3}, x_{4}, x_{5}\right) \rightarrow\left(\alpha x_{1}, \alpha x_{2}, x_{3}, x_{4}, x_{5}\right)
$$

where $\alpha^{2}=1$, and thus the $\mathbb{Z}_{2}$ is seen to be part of the projective equivalence identification,

$$
\begin{gathered}
\mathbb{P}_{(1,7,2,2,2)}=\mathbb{C}_{5} / \sim \\
-15-
\end{gathered}
$$


with $\left(x_{1}, x_{2}, x_{3}, x_{4}, x_{5}\right) \sim\left(\lambda x_{1}, \lambda^{7} x_{2}, \lambda^{2} x_{3}, \lambda^{2} x_{4}, \lambda^{2} x_{5}\right)$, where $\lambda \in \mathbb{C}^{\star}$.

Suppose we add the fundamental deformation and one more to $P_{0}$, so that $P=$ $P_{0}-\phi_{0} x_{1} x_{2} x_{3} x_{4} x_{5}-\phi_{1} x_{1}^{6} x_{3}^{2} x_{4}^{2}$. Then the periods in the coordinate patch where $x_{5}=1$ are

$$
\begin{aligned}
\varpi^{(5)} & =\frac{C_{x}}{2^{2} \cdot 7^{3}} \sum_{m=0}^{\infty} \sum_{n=0}^{\infty} e^{2 \pi i\left(\frac{m+6 n+1}{14} \delta_{1}+\frac{m+2 n+1}{7}\left(\delta_{3}+\delta_{4}\right)\right)} \frac{\phi_{0}^{m}}{m !} \frac{\phi_{1}^{n}}{n !} \\
& \times\left(1-e^{2 \pi i\left(\frac{m+6 n+1}{14}\right)}\right)\left(1-e^{2 \pi i\left(\frac{m+1}{2}\right)}\right)\left(1-e^{2 \pi i\left(\frac{m+2 n+1}{7}\right)}\right)^{2} \\
& \times \Gamma\left(\frac{m+6 n+1}{14}\right) \Gamma\left(\frac{m+1}{2}\right) \Gamma^{2}\left(\frac{m+2 n+1}{7}\right) \Gamma\left(\frac{m+1}{7}\right) .
\end{aligned}
$$

We need only vary $\delta_{1}$ and $\delta_{3}$ to find all the periods.

Suppose we add the images of these deformations under (3.14) to $\tilde{P}_{0}$, so that $\tilde{P}=$ $\tilde{P}_{0}-\psi_{0} y_{1} y_{2} y_{3} y_{4} y_{5}-\psi_{1} y_{1}^{3} y_{3}^{2} y_{4}^{2}$. Then the periods in the coordinate patch where $y_{5}=1$ are

$$
\begin{aligned}
\tilde{\varpi}^{(5)} & =\frac{C_{y}}{2 \cdot 7^{3}} \sum_{m=0}^{\infty} \sum_{n=0}^{\infty} e^{2 \pi i\left(\frac{m+6 n+1}{14} \delta_{1}+\frac{m+2 n+1}{7}\left(\delta_{3}+\delta_{4}\right)\right)} \frac{\psi_{0}^{m}}{m !} \frac{\psi_{1}^{n}}{n !} \\
& \times\left(1-e^{2 \pi i\left(\frac{m+6 n+1}{14}\right)}\right)\left(1-e^{2 \pi i\left(\frac{m+1}{2}\right)}\right)\left(1-e^{2 \pi i\left(\frac{m+2 n+1}{7}\right)}\right)^{2} \\
& \times \Gamma\left(\frac{m+6 n+1}{14}\right) \Gamma\left(\frac{m+1}{2}\right) \Gamma^{2}\left(\frac{m+2 n+1}{7}\right) \Gamma\left(\frac{m+1}{7}\right) .
\end{aligned}
$$

These are the same as (3.19) up to a normalization factor, letting $\phi_{i}=\psi_{i}$. This is not surprising - the period calculations are related simply by the change of variables (3.14).

The value of this comes when we want to look at the dependence of the periods of $\mathcal{M}$ on twisted moduli. This we can do by looking at the dependence of the periods of $\tilde{\mathcal{M}}$ on the deformations (3.15). So suppose $\tilde{P}=\tilde{P}_{0}-\psi_{0} y_{1} y_{2} y_{3} y_{4} y_{5}-\psi_{2} y_{2} y_{3}^{4}$. Then the periods in the coordinate patch where $y_{5}=1$ are

$$
\begin{aligned}
\tilde{\varpi}^{(5)} & =\frac{C_{y}}{2 \cdot 7^{3}} \sum_{m=0}^{\infty} \sum_{n=0}^{\infty} e^{2 \pi i\left(\frac{m-n+1}{14} \delta_{1}+\frac{m+4 n+1}{7} \delta_{3}+\frac{m+1}{7} \delta_{4}\right)} \frac{\psi_{0}^{m}}{m !} \frac{\psi_{2}^{n}}{n !} \\
& \times\left(1-e^{2 \pi i\left(\frac{m+n+1}{2}\right)}\right)\left(1-e^{2 \pi i\left(\frac{m-n+1}{14}\right)}\right)\left(1-e^{2 \pi i\left(\frac{m+4 n+1}{7}\right)}\right)\left(1-e^{2 \pi i\left(\frac{m+1}{7}\right)}\right) \\
& \times \Gamma\left(\frac{m+n+1}{2}\right) \Gamma\left(\frac{m-n+1}{14}\right) \Gamma\left(\frac{m+4 n+1}{7}\right) \Gamma^{2}\left(\frac{m+1}{7}\right) .
\end{aligned}
$$

Reinterpreted as periods of $\mathcal{M}$, the $\psi_{2}$ dependence is the dependence on the modulus associated to a twisted state, i.e., one of the states in (3.17). Note that the same calculation could have been done by adding the deformations in (3.16) to $\tilde{P}_{0}$. 


\subsection{A Higher Dimensional Example}

Let us consider the following example: $\mathbb{C}_{1,6,6,4,4,6,9}^{7}[18]$, the zero locus of a defining polynomial of degree 18 . Take the polynomial to be

$$
P_{\phi}=x_{0}^{18}+x_{1}^{3}+x_{2}^{3}+x_{1} x_{3}^{3}+x_{2} x_{4}^{3}+x_{5}^{3}+x_{6}^{2}-\phi_{0} x_{0}^{4} x_{3} x_{4} x_{5}-\phi_{1} x_{0}^{6} x_{1} x_{2} .
$$

Following the results of section 2 and the discussion above it is now straightforward to write down the periods associated to the above family. In patch $\mathcal{U}_{0}$ we have

$$
\begin{aligned}
\varpi_{\left(\delta_{1}, \ldots, \delta_{6}\right)=} & C \int_{\left(\delta_{1}, \ldots, \delta_{6}\right)} \frac{\prod_{i=1}^{6} \mathrm{~d} x_{i}}{\left(\left.P_{\phi}\right|_{x_{0}=1}\right)^{2}} \\
= & C \sum_{n, m=0}^{\infty} \frac{\phi_{0}^{n}}{n !} \frac{\phi_{1}^{m}}{m !} \int_{0}^{\infty} \mathrm{d} s e^{-s} s^{n+m+1} \\
& \times \int_{\left(\delta_{1}, \ldots, \delta_{6}\right)} \mathrm{d}^{6} x e^{-s\left(x_{1}^{3}+x_{1} x_{3}^{3}+x_{2}^{3}+x_{2} x_{4}^{3}+x_{5}^{3}+x_{6}^{2}\right)} x_{1}^{m} x_{2}^{m} x_{3}^{n} x_{4}^{n} x_{5}^{n} \\
= & C \frac{\Gamma(1 / 2)}{3^{5}} \sum_{n, m=0}^{\infty} \frac{\phi_{0}^{n}}{n !} \frac{\phi_{1}^{m}}{m !} e^{2 \pi i\left(\left(\sum_{i=3}^{5} \delta_{i}\right)\left(\frac{n+1}{3}\right)+\left(\delta_{1}+\delta_{2}\right)\left(\frac{3(m+1)-(n+1)}{9}\right)\right)} \\
& \times\left(1-e^{2 \pi i\left(\frac{n+1}{3}\right)}\right)^{3}\left(1-e^{2 \pi i\left(\frac{3(m+1)-(n+1)}{9}\right)}\right)^{2} \\
& \times \Gamma^{3}\left(\frac{n+1}{3}\right) \Gamma^{2}\left(\frac{3(m+1)-(n+1)}{9}\right) \Gamma\left(\frac{6 m+4 n+1}{18}\right) .
\end{aligned}
$$

Note that the period depends only on combinations $\delta_{3}+\delta_{4}+\delta_{5}, \delta_{1}+\delta_{2}$ and that letting $\delta_{3} \rightarrow \delta_{3}+1$ is equivalent to $\delta_{1} \rightarrow \delta_{1}+6$. Thus we may set $\delta_{2}=\ldots=\delta_{5}=0$ if we allow $\delta_{1}$ to take values $0,1, \ldots, 8$. By rearranging the periods using (3.3) we finally obtain:

$$
\varpi_{\left(\delta_{1}\right)}^{(0)}=C \frac{\Gamma(1 / 2)(2 \pi i)^{5}}{3^{5} \phi_{0} \phi_{1}} \sum_{m, n=1}^{\infty} \frac{e^{2 \pi i m\left(\frac{\delta_{1}+1}{3}\right)} e^{2 \pi i n\left(\frac{7-2 \delta_{1}}{18}\right)} \phi_{0}^{n} \phi_{1}^{m} \Gamma\left(\frac{6 m+4 n-9}{18}\right)}{\Gamma(n) \Gamma(m) \Gamma^{3}\left(1-\frac{n}{3}\right) \Gamma^{2}\left(1-\frac{3 m-n}{9}\right)} .
$$

The $\delta_{1}$ would generate nine periods but they are not all independent. Rather, because of the factor $\Gamma^{3}\left(1-\frac{n}{3}\right)$ in the denominator in $\varpi_{\left(\delta_{1}\right)}^{(0)}$ there are the following relations:

$$
\sum_{j=0,1,2} \varpi_{3 j+k}=0, \quad k=0,1,2
$$

Thus we are left with six linearly independent periods as expected, since we are considering a model with two complex structure deformations. 


\section{Acknowledgments:}

The authors acknowledge fruitful discussions with $\mathrm{P}$. Candelas and X. de la Ossa. E. D. would like to thank $R$. Schimmrigk for discussions of fractional transformations. P. B. was supported by the American-Scandinavian Foundation, the Fulbright Program, NSF grants PHY 8904035 and PHY 9009850, DOE grant DE-FG02-90ER4052 and the Robert A. Welch Foundation. P. B. would also like to thank the ITP, Santa Barbara and the Theory Division, CERN for their hospitality during the initial stages of this project. T.H. was supported by the Howard University '93 FRSG Program. E. D. and D. J. were supported by NSF grant PHY 9009850 and the Robert A. Welch Foundation.

\section{Appendix A. Turning Spokes into Cycles}

Consider a Fermat-type polynomial of weight $d$, and suppose the weight of coordinate $x_{1}$ is 1. Take a polynomial

$$
p=x_{1}^{d}+x_{2}^{a_{2}}+x_{3}^{a_{3}}+x_{4}^{a_{4}}+x_{5}^{a_{5}}-\psi x_{1} x_{2} x_{3} x_{4} x_{5}
$$

Now the symmetries of this polynomial include the following, listed along with their actions ${ }^{2}$ :

$$
\begin{aligned}
& g_{1}=\left(\mathbb{Z}_{d}: 1, k_{2}, k_{3}, k_{4}, k_{5}\right), \\
& g_{2}=\left(\mathbb{Z}_{a_{2}}: a_{2}-1,1,0,0,0\right), \\
& g_{3}=\left(\mathbb{Z}_{a_{3}}: a_{3}-1,0,1,0,0\right), \\
& g_{4}=\left(\mathbb{Z}_{a_{4}}: a_{4}-1,0,0,1,0\right), \\
& g_{5}=\left(\mathbb{Z}_{a_{5}}: a_{5}-1,0,0,0,1\right) .
\end{aligned}
$$

The inhomogeneous coordinates in the patch $\mathcal{U}_{l}(l \neq 1)$ are

$$
\xi_{i}^{(l) \stackrel{\text { def }}{=}}\left(\frac{x_{i}}{x_{l}^{k_{i} / k_{l}}}\right) \text { in } \mathcal{U}_{l}, \text { where } x_{l} \neq 0
$$

The building blocks for the cycles are three-chains, written in any patch $\mathcal{U}_{l}(l \neq 1)$ as

$$
\begin{aligned}
V_{j}^{(l)}= & \left\{\xi_{k}^{(l)} \mid \xi_{l}^{(l)}=1 ; \xi_{i}^{(l)} \text { real and positive, for } i \neq 1, l\right. \\
& \left.\xi_{1}^{(l)} \text { is a solution of } p=0 \text { on the branch } \arg \left(\xi_{1}^{(l)}\right) \rightarrow \pi+\frac{2 \pi j}{d} \text { as } \psi \rightarrow 0\right\} .
\end{aligned}
$$

\footnotetext{
2 We will use the notation $\left(\mathbb{Z}_{k}: \Theta_{1}, \ldots, \Theta_{5}\right)$ for a $\mathbb{Z}_{k}$ symmetry with the action $\left(X_{1}, \ldots, X_{5}\right) \rightarrow\left(\alpha^{\Theta_{1}} X_{1}, \ldots, \alpha^{\Theta_{5}} X_{5}\right)$, where $\alpha^{k}=1$.
} 
The three-chain on the manifold is then $V_{j}=U V_{j}^{(l)}$.

In a patch $\mathcal{U}_{l}$ there are 3 two-dimensional boundaries to $V_{j}^{(l)}$, which we denote $B_{j, i}^{(l)}, \quad i=2,3,4$. These occur where one of the $\xi_{i}^{(l)}$ vanishes and the other two do not. On the overlap of two patches,

$$
B_{j, i}^{(l)}=B_{j, i}^{(m)} \quad \text { if } \quad i \neq l \neq m
$$

Note that $B_{j, i}^{(l)}$ is not in the patch $\mathcal{U}_{i}$. Over the manifold, then, there are four independent components to the boundary of $V_{j}$ :

$$
\begin{aligned}
& B_{j, 2}=B_{j, 2}^{(3)}=B_{j, 2}^{(4)}=B_{j, 2}^{(5)}, \\
& B_{j, 3}=B_{j, 3}^{(2)}=B_{j, 3}^{(4)}=B_{j, 3}^{(5)}, \\
& B_{j, 4}=B_{j, 4}^{(2)}=B_{j, 4}^{(3)}=B_{j, 4}^{(5)}, \\
& B_{j, 5}=B_{j, 5}^{(2)}=B_{j, 5}^{(3)}=B_{j, 5}^{(4)} ;
\end{aligned}
$$

only three will be manifest in any patch.

Let us restrict to patch $\mathcal{U}_{5}$, and momentarily drop the superscript labelling the patch. Now if $A$ is the operation that takes $\psi \rightarrow e^{-\frac{2 \pi i}{d}} \psi$, then certain powers of $A$ are equivalent to a coordinate transformation

$$
A^{k_{j}}: x_{j} \rightarrow e^{\frac{2 \pi i k_{j}}{d}} x_{j}
$$

The integration contours using adjacent spokes in $\xi_{2}, \xi_{3}$, and $\xi_{4}$ are therefore formed by

$$
Q=\left(1-A^{k_{2}}\right)\left(1-A^{k_{3}}\right)\left(1-A^{k_{4}}\right) V_{j}
$$

Note that $Q$ has no boundary of the form $B_{m, 4}$ owing to the fact that $\left(1-A^{k_{4}}\right) B_{j, 4}=0$. Similarly, all the $B_{m, 2}$ and $B_{m, 3}$ components vanish, so $Q$ is indeed a cycle.

In a quotient by (A.2) (say, for construction of the mirror model), the boundaries $B_{m, 2}$ 's become identified, and similarly the $B_{m, 3}$ 's, and so on. Then we may write

$$
\begin{aligned}
Q & =\left(1-A^{k_{2}}\right)\left(1-A^{k_{3}}\right)\left(1-A^{k_{4}}\right) V_{j} \\
& =V_{j}-V_{j+k_{2}}-V_{j+k_{3}}-V_{j+k_{4}}+V_{j+k_{2}+k_{3}}+V_{j+k_{2}+k_{4}}+V_{j+k_{3}+k_{4}}-V_{j+k_{2}+k_{3}+k_{4}} .
\end{aligned}
$$

A similar approach may be used to show that the relevant contours for the other polynomial types are cycles as well. In particular, any polynomial can be put in a Fermat form by fermatization, as described in section 2.5 . 


\section{References}

[1] T. Hübsch: Calabi-Yau Manifolds-A Bestiary for Physicists (World Scientific, Singapore, 1992).

[2] P. Candelas, P.S. Green and T. Hübsch: Nucl. Phys. B330 (1990) 49.

[3] A. Strominger: Commun. Math. Phys. 133 (1990) 163.

[4] P. Candelas and X. C. de la Ossa: Nucl. Phys. B355 (1991) 455.

[5] J. Distler and B.R. Greene: Nucl. Phys. B309 (1988) 295.

[6] P. Candelas: Nucl. Phys. B298 (1988) 458.

[7] P. Berglund, T. Hübsch and L. Parkes: Commun. Math. Phys. 148 (1992) 57.

[8] P. Berglund and T. Hübsch: "Couplings for Compactification", Howard University preprint HUPAPP-93/2, Nucl. Phys. B (in press).

[9] P. Berglund and T. Hübsch: Nucl. Phys. B393 (1993) 377, also in Essays on Mirror Manifolds, p.388, ed. S.-T. Yau, (Intl. Press, Hong Kong, 1992).

[10] P. Candelas, X. de la Ossa, P. Green and L. Parkes: Nucl. Phys. B359 (1991) 21.

[11] P. Berglund, P. Candelas, X. de la Ossa, A. Font, T. Hübsch, D. Jančić and F. Quevedo: Periods for Calabi-Yau and Landau-Ginzburg Vacua, CERN preprint CERN-TH $6865 / 93$.

[12] P. Candelas, E. Derrick and L. Parkes: "Generalized Calabi-Yau Manifolds and the Mirror of a Rigid Manifold", Nucl. Phys. B407 (1993) 115.

[13] S. Cecotti and C. Vafa: Topological Anti-Topological Fusion. Nucl. Phys. B367 (1991) 359-461.

[14] T. Hübsch: Commun. Math. Phys. 108 (1987) 291.

[15] P. Green and T. Hübsch: Commun. Math. Phys. 109 (1987) 99.

[16] P. Candelas, A.M. Dale, C. A. Lütken and R. Schimmrigk: Nucl. Phys. B298 (1988) 493.

[17] B. Greene, S.-S. Roan and S.-T. Yau: Commun. Math. Phys. 142 (1991)245.

[18] M. Atiyah, R. Bott and L. Gårding: Acta Math. 131 (1973) 145.

[19] P. Candelas, M. Lynker and R. Schimmrigk: Nucl. Phys. B341 (1990) 383.

[20] A. Erdélyi, F. Oberhettinger, W. Magnus and F. G. Tricomi: Higher Transcendental Functions, 3 Vols. (McGraw-Hill, New York, 1953). 
[21] V.I. Arnold: in Proceedings of the International Congress of Mathematicians, p.19, Vancouver, 1974, Singularity Theory (London Math. Soc. Lecture Note Series 53, Cambridge University Press, Cambridge, 1981);

V.I. Arnold, S.M. Gusein-Zade and A.N. Varchenko: Singularities of Differentiable Maps (Birkhäuser, Boston, 1985).

[22] M. Kreuzer and H. Skarke: "On the Classification of Quasihomogenous Functions", CERN preprint CERN-TH-6373/92.

[23] M. Kreuzer and H. Skarke: Nucl. Phys. B388 (1992) 113.

[24] A. Klemm and R. Schimmrigk: "Landau-Ginzburg Vacua", CERN preprint CERNTH-6459/92, Universität Heidelberg report HD-THEP-92-13.

[25] M. Lynker and R. Schimmrigk: Phys. Lett. 249B (1990) 237.

[26] C. Vafa and N.P. Warner: Phys. Lett. 218B (1989) 377;

E.Martinec: Phys. Lett. B217 (1989) 431. 